\title{
Treatment of osteoporosis with eldecalcitol, a new vitamin $D$ analog: a comprehensive review and meta-analysis of randomized clinical trials
}

This article was published in the following Dove Press journal:

Drug Design, Development and Therapy

28 January 2016

Number of times this article has been viewed

\author{
Zhixing $\mathrm{Xu}^{\prime}$ \\ Changchun Fan ${ }^{2}$ \\ Xuechun Zhao ${ }^{3}$ \\ Hairong Tao' \\ 'Department of Orthopedic Surgery, \\ The Third People's Hospital Affiliated \\ to Shanghai Jiaotong University \\ School of Medicine, Shanghai, People's \\ Republic of China; ${ }^{2}$ Department of \\ Orthopedic Surgery, Jinan Military \\ General Hospital, ${ }^{3}$ Department of \\ Orthopedic Surgery, The Third \\ Hospital of Jinan City, Jinan, People's \\ Republic of China
}

Objective: Eldecalcitol (ELD) is an active form of vitamin D analog that has been approved for the treatment of osteoporosis in Japan. Over recent years, a number of multicenter, randomized controlled clinical trials have been conducted. Our goal is to comprehensively summarize the results from these studies.

Methods: We searched the databases MEDLINE, EMBASE, and Cochrane Central Register of Controlled Trials up to February 28, 2015. Each database was searched using search terms "Eldecalcitol" and "ED-71" and the results were combined. The retrieved data from three independent clinical trials included a total of 1,332 patients with osteoporosis. After the data were pooled from three trials, RevMan software was used to conduct meta-analyses to determine the effects of ELD on bone mineral density (BMD) and bone turnover marker (BTM) type I collagen amino-terminal telopeptide (NTX). Effects of ELD on some of the bone formation and bone resorption parameters, incidence of vertebral fractures at the lower spine, and health-related quality of life (HRQOL) in patients with osteoporosis were also summarized.

Results: With a test for overall effect $Z=6.35$, ELD could increase lumbar BMD $(P<0.00001)$. In comparison with alphacalcidol, ELD suppressed the NTX level to a greater degree (test for overall effect $Z=3.82, P<0.0001$ ). ELD was also found to suppress bone alkaline phosphatase (BALP) by $19 \%(P<0.01)$ and osteocalcin by $19 \%(P<0.01)$ at the dose of $0.75 \mu \mathrm{g} / \mathrm{day}$. Compared to alfacalcidol, ELD showed higher potency in suppressing serum BALP (26 \pm 9 vs $32 \pm 11 \mathrm{U} / \mathrm{L}, P<0.05)$ and amino-terminal propeptide of procollagen I (PINP) (42 \pm 15 vs $59 \pm 23$ $\mathrm{ng} / \mathrm{mL}, P<0.05)$. In addition, ELD was found to be more effective in reducing the incidence of vertebral fractures at the lower spine $(P=0.029)$.

Conclusion: Our meta-analysis showed that ELD was more potent than alphacalcidol in reducing BTM (NTX). Clinical data together suggest that ELD is efficient in treating osteoporosis by increasing lumbar BMD; suppressing BTMs, including NTX, BALP, osteocalcin, and PINP; resulting in the reduction in the incidence of vertebral fractures at the lower spine; and increasing the HRQOL in patients with osteoporosis.

Keywords: BMD, NTX, BALP, PINP

\section{Introduction}

Osteoporosis is a progressive systemic skeletal disease characterized by low bone mass and micro-architectural deterioration of bone tissue, with a consequent increase in bone fragility and susceptibility to fracture. ${ }^{1}$ Changes in bone turnover with aging are responsible for bone loss and play a major role in osteoporosis. An imbalance in bone turnover impacts on bone strength as a result of reductions in bone volume and mineralization, loss of trabeculae, deterioration of trabecular connectivity, and the formation of resorption cavities and trabecular perforations. Therefore, an increase
Correspondence: Hairong Tao

Department of Orthopedic Surgery, The Third People's Hospital Affiliated to Shanghai Jiaotong University School of Medicine, Shanghai, People's Republic of China

Email hairongtao@yeah.net 
in bone turnover where resorption exceeds formation is not only inversely correlated with bone mineral density (BMD), but may also alter bone architecture and porosity, increasing the risk of fracture beyond that due to reduced BMD, and can therefore be an independent predictor of fracture risk. ${ }^{2-5}$

Bone density scanning using dual-energy X-ray absorptiometry is currently the most commonly used means of measuring BMD. It is typically used to diagnose and follow osteoporosis. The World Health Organization (WHO) has established criteria for making the diagnosis of osteoporosis, as well as determining levels that predict higher chances of fractures. These criteria are based on comparison of the BMD of the patient to that of a typical healthy, young female. BMD values that fall 2.5 standard deviations below the average for the healthy, young female's BMD are diagnosed as osteoporotic. BMD values less than the healthy, young female, but not 2.5 standard deviations below the average are referred to as osteopenic. In addition to serial BMD measurements, biochemical markers of bone turnover are also widely used to monitor treatment response, since they are noninvasive and relatively cheap compared with dual-energy X-ray absorptiometry.

Formation markers are products from the action of osteoblasts. These include bone-specific alkaline phosphatase (BALP), procollagen type 1 amino-terminal propeptide (PINP), osteocalcin (or bone GLA-protein), and procollagen type 1 carboxy-terminal propeptide (P1CP). Resorption markers are products from the action of osteoclasts. These include carboxy-terminal telopeptide cross-linked type 1 collagen (CTX), type I collagen amino-terminal telopeptide (NTX), and urine deoxypyridinoline.

Vitamin D metabolism plays a critical role in the regulation of mineral and bone homeostasis. ${ }^{6}$ The active form of vitamin $\mathrm{D}, 1 \alpha, 25$-dihydroxyvitamin $\mathrm{D} 3$ (1 $\alpha, 25-(\mathrm{OH}) 2 \mathrm{D} 3)$, acts through the vitamin $\mathrm{D}$ receptor (VDR) present on target cells such as osteoblasts and osteoclasts ${ }^{7,8}$ to stimulate calcium absorption and reabsorption while blocking both the synthesis and secretion of another essential regulator of mineral balance, the parathyroid hormone (PTH). ${ }^{9}$ At least two mechanisms are involved in vitamin D-mediated regulation of mineral and bone homeostasis: first, it binds to the VDR on osteoblastic cells and regulates osteoclastic activity via the osteoprotegerin (OPG)/receptor activator nuclear factor kB (RANK) system; ${ }^{10}$ second, it secures a supersaturated state of calcium-phosphorus products in the blood, enabling osteoid mineralization. ${ }^{11}$ Due to its calcemic activity and its potential role in promoting osteoclastic bone resorption, the use of vitamin D in the treatment of osteoporosis has been hindered. ${ }^{12-14}$ However, much effort has been made to synthesize compounds that would retain only the differentiation and antiproliferative effects of $1 \alpha, 25-(\mathrm{OH}) 2 \mathrm{D} 3$ and allow for safer usage and less side effects. ${ }^{15,16}$ In fact, the active form of vitamin D (1 $\alpha, 25$-dihydroxyvitamin D3, calcitriol) and its prodrug ( $1 \alpha$-hydroxyvitamin D3, alfacalcidol) have been approved for the treatment of osteoporosis in Japan. ${ }^{17}$ Both calcitriol and alfacalcidol have been shown to increase $\mathrm{BMD}^{18,19}$ and reduce the risk of vertebral and nonvertebral fractures. ${ }^{20-22}$ Eldecalcitol (ELD), formerly known as ED-71 that was developed in the early 1980s, is an analog of $1 \alpha, 25-(\mathrm{OH}) 2 \mathrm{D} 3$ bearing a hydroxypropyloxy residue at the $2 \beta$ position. In a rat ovariectomized model of osteoporosis, ELD has been demonstrated to lower biochemical and histological parameters of bone resorption. ${ }^{23}$ Further study showed that these effects on bone were observed without sustained hypercalcemia or hypercalciuria. ${ }^{24}$ ELD has also been approved as a drug for treatment of osteoporosis in Japan. In the recent years, a few clinical trials have been conducted to examine the efficacy of ELD in treating osteoporosis. This study was aimed to summarize the results of these clinical trials and evaluating the clinical effects of ELD on lumbar and hip BMD, and bone turnover markers (BTMs) in patients with osteoporosis.

\section{Methods}

\section{Literature search and study selection}

Databases MEDLINE, EMBASE, and the Cochrane Central Register of Controlled Trials were searched till February 18, 2015 to identify all potential clinical trials using ELD for the treatment of osteoporosis. We performed the literature search using the following search term: (eldecalcitol OR ED-71). After excluding review articles, basic studies, animal studies, pharmacokinetic studies, articles in languages other than English, and conference abstracts, we identified a total of eleven clinical studies investigating the efficacy of ELD in treatment of osteoporosis. If the same study had been published in different articles, we selected the most recent publication or the publication covering most detailed information. Literature search was repeated using the same databases and search terms during the revision of this article and no new study was found.

\section{Data extraction and quality assessment}

For the meta-analysis study, we chose lumber BMD, hip BMD, and BTM NTX as the outcome measures. Data on severe vertebral fractures, BTMs BALP, osteocalcin, NTX, and serum PINP were also extracted for summary 
and discussion. Original data collection forms were used to abstract the following information from single studies: sample size, dose, and changes from base line. Two reviewers screened articles independently, and disagreements were resolved through discussion. The Cochrane Collaboration's tool was used to assess risk of bias.

\section{Evaluation of heterogeneity and statistics}

RevMan software, version 5.3, was used for the calculation of heterogeneity parameter, quantitative meta-analyses, and generation of forest plots. The $I^{2}$ value was calculated to determine the degree of heterogeneity. An $I^{2}$ value from $50 \%$ to $75 \%$ represented moderate heterogeneity, and an $I^{2}$ value above $75 \%$ was defined as high heterogeneity. In the presence of high heterogeneity, the random effect model was implemented. Risk ratios and their $95 \%$ confidence intervals (CIs) were calculated.

\section{Results}

We identified 123 entries in MEDLINE, 176 entries in EMBASE, and 20 entries in Cochrane Central Register of Controlled Trials. As is shown in Figure 1, a total of eleven studies were identified and analyzed. Among these studies, nine came from three independent trials using ELD as monotherapy, involving 1,332 patients who were given placebo $(n=53)$, alfacalcidol $(n=546)$, or ELD $(n=734)$. The first trial published in 2005 tested the effects of different doses of ELD on lumbar BMD, total hip BMD, and BTMs, including BALP, osteocalcin, NTX, BALP, and PINP. ${ }^{24}$ The second trial published in 2010 compared between alfacalcidol and ELD the effects on BTMs BALP and NTX. ${ }^{25}$ The third trial also compared the effects of alfacalcidol and ELD and the results were reported in multiple articles. In the report published in 2011, the effects of alfacalcidol and ELD on lumbar BMD and hip BMD were reported. ${ }^{26}$ In the report published in 2012, their effects on NTX, BALP, and PINP were revealed. ${ }^{27}$ The main features and their quantitative data synthesis are summarized in Table 1. Not all studies were included in Table 1, since some of them basically analyzed the same data from different angles.

The risk of bias assessment for these trials is presented in Table 2. Trials 1, 2, and 3 were found to be at moderate, high, and low risk of bias, respectively. The common problem for all the three trails was inadequate description of the randomization method and the overall risk of bias for all the trials was rated as moderate. The other two trials used ELD as a component in combination therapies. Results from these trials were excluded from our meta-analysis.

Among all parameters reported, only lumbar BMD, hip BMD, and NTX were examined in at least two independent trials. In the second trial, ELD increased the lumber and hip BMD by $3.1 \pm 0.7$ and $0.9 \pm 0.5$, respectively. ${ }^{24}$ In the third trial, alfacalcidol increased the lumbar BMD by $0.2 \pm 0.4$ but decreased hip BMD by $2.4 \pm 0.3$, and ELD increased the lumber and hip BMD by $3.5 \pm 0.2$ and $0.4 \pm 0.2$, respectively. ${ }^{26}$ Our meta-analyses found that at dose not less than $0.75 \mu \mathrm{g} / \mathrm{day}$, ELD was able to significantly increase lumbar BMD with test for overall effect $Z=6.35(P<0.00001)($ HT $P<0.00001$,

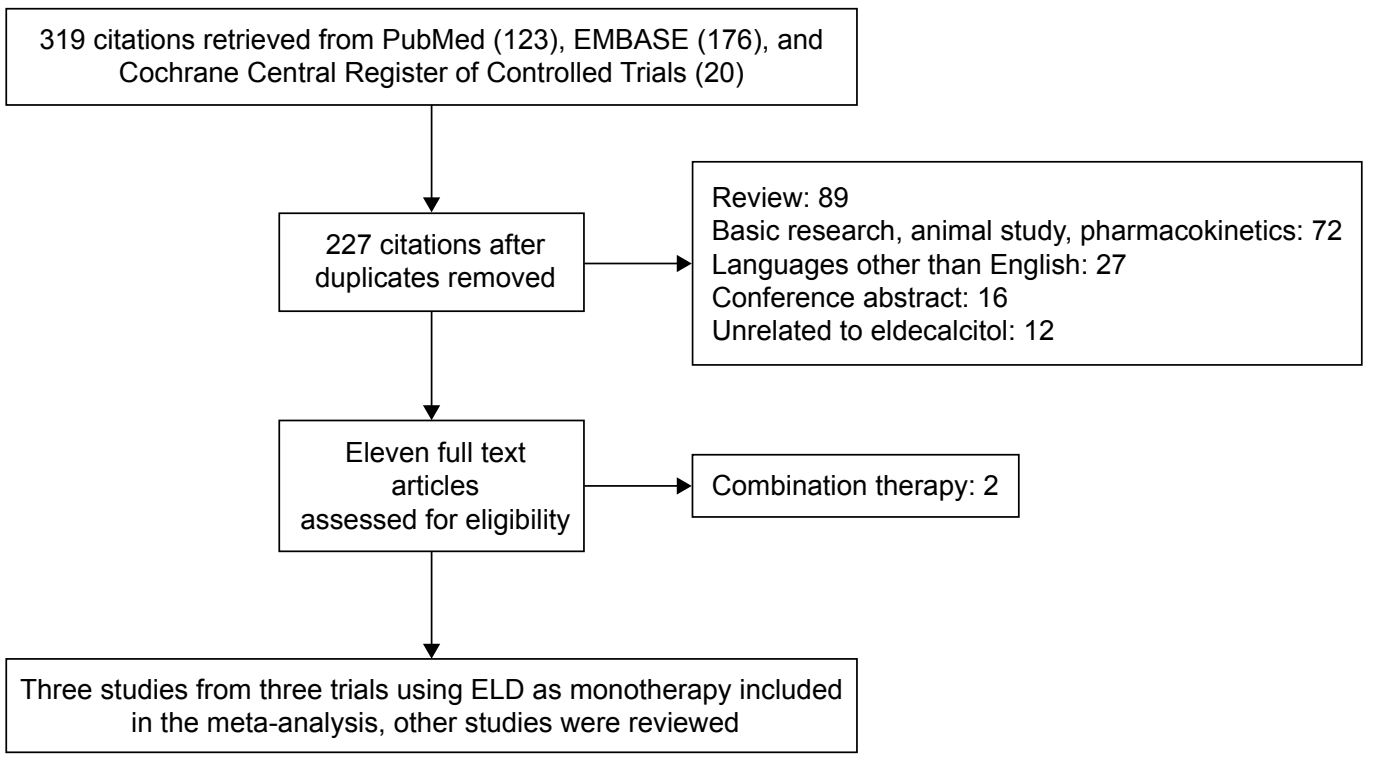

Figure I Flow diagram for study selection. Abbreviation: ELD, eldecalcitol. 


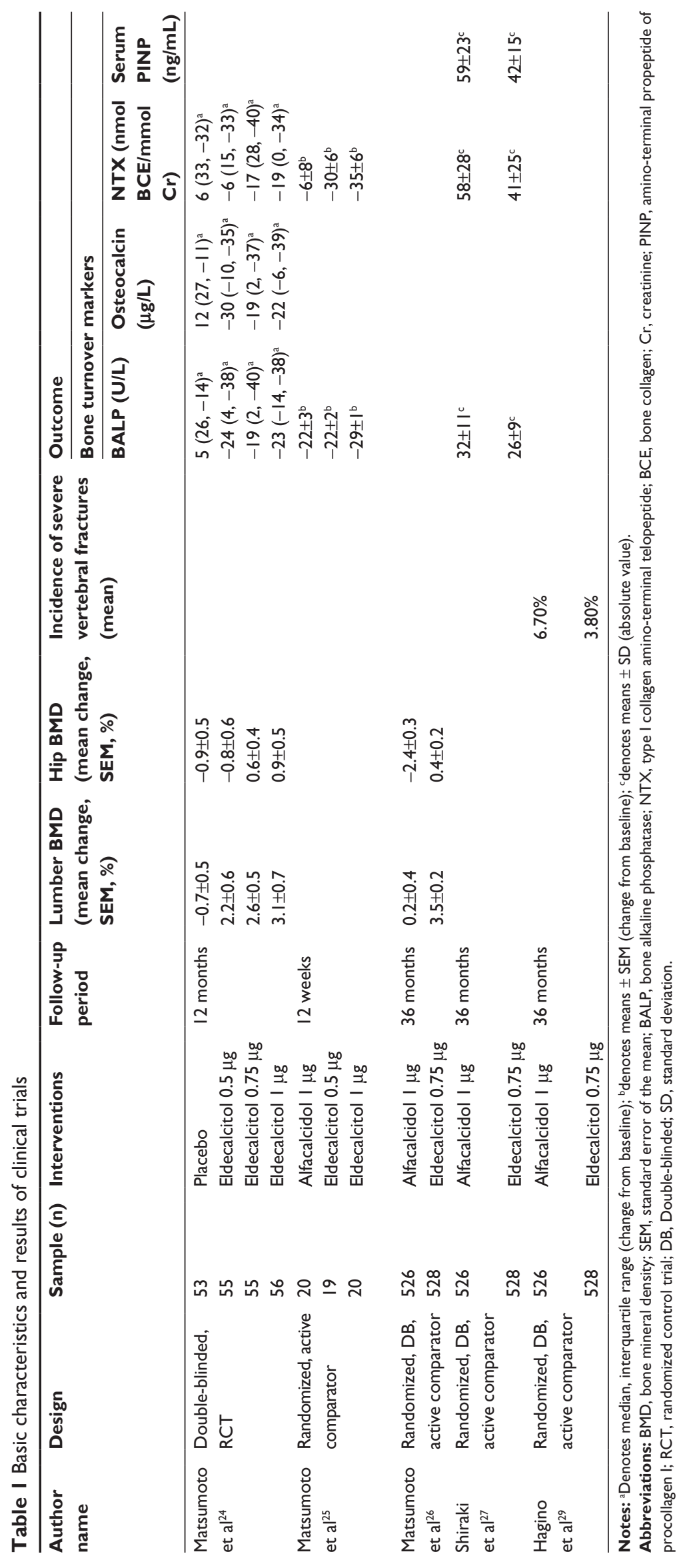


Table 2 Assessment of risk of bias by The Cochrane Collaboration's tool

\begin{tabular}{|c|c|c|c|}
\hline Type of risk of bias & Trial I & Trial 2 & Trial 3 \\
\hline Random sequence generation (selection bias) & Unclear & Unclear & Unclear \\
\hline Allocation concealment (selection bias) & Unclear & No & Yes \\
\hline Blinding of outcome assessment (detection bias) & Yes & Unclear & Yes \\
\hline Incomplete outcome data (attrition bias) & Yes & Unclear & Yes \\
\hline Selective reporting (reporting bias) & Yes & Unclear & Yes \\
\hline
\end{tabular}

$\tau^{2}=0.40, \chi^{2}=58.5, d f=1, I^{2}=98 \%$ ) (Figure 2). Edecalcitol did not seem to increase total hip BMD though, with test for overall effect $Z=1.00(P=0.32)$ (Heterogeneity [HT] $P<0.00001$, $\tau^{2}=3.92, \chi^{2}=831.55, d f=1, I^{2}=100 \%$ ) (Figure 3).

All the three trials examined the effects of ELD on BTM NTX. In the first trial involving 219 osteoporotic patients, $1 \mu \mathrm{g} /$ day of edecalcitol decreased urinary NTX from the baseline with a median percentage of $19 \%(P<0.01) \cdot{ }^{24}$ In the second trial involving 59 Japanese postmenopausal women, suppression of urinary NTX was much stronger in both 0.5 and $1 \mu \mathrm{g}$ ELD-treated groups than in $1 \mu \mathrm{g}$ alfacalcidol-treated subjects, with urinary NTX decreasing by $6 \%, 30 \%$, and $35 \%$ from the baseline, respectively. ${ }^{25}$ In the third trial involving 1,054 patients, ELD-treated group $(0.75 \mu \mathrm{g} /$ day $)$ was found to have lower urinary NTX than alfacalcidol-treated group $\left(1 \mu \mathrm{g} /\right.$ day) $(41 \pm 25 \mathrm{nmol}$ vs $58 \pm 28 \mathrm{nmol}, P<0.05) .{ }^{27}$ Since the effects of NTX were reported as medians of changes from the baseline in the first trial, the second and third trials reported only the means of NTX. Therefore, we carried out the meta-analysis for NTX using data only from the second and third trials, and the result, with test for overall effect $Z=3.82(P=0.0001)\left(\right.$ HT $P<0.0001, \tau^{2}=68.16, \chi^{2}=18.76$, $\left.d f=1, I^{2}=95 \%\right)$, suggested that ELD suppressed NTX to a greater degree when compared to alfacalcidol (Figure 4).

In addition to NTX, some other BTMs were also investigated in these studies. In the first trial, ELD was found to suppress BALP with median percentages of $24 \%(P<0.01)$, $19 \%(P<0.01)$, and 23\% $(P<0.01)$ and osteocalcin with median percentages of $30 \%(P<0.01), 19 \%(P<0.01)$, and $22 \%(P<0.01)$. In the third trial, $0.75 \mu \mathrm{g} /$ day of edecalcitol

\begin{tabular}{|c|c|c|c|c|c|c|c|c|}
\hline Study or subgroup & $\begin{array}{l}\text { Eldeca } \\
\text { Mean }\end{array}$ & $\begin{array}{l}\text { Icitol } \\
\text { SD }\end{array}$ & Total & $\begin{array}{l}\text { Contrc } \\
\text { Mean }\end{array}$ & SD & Total & Weight & $\begin{array}{l}\text { Mean difference } \\
\text { IV, random, } 95 \%\end{array}$ \\
\hline Matsumoto et $\mathrm{al}^{24}$ & 3.1 & 0.7 & 56 & 0.7 & 0.5 & 53 & $49.2 \%$ & $2.40(2.17,2.63)$ \\
\hline Matsumoto et $\mathrm{al}^{26}$ & 3.5 & 0.2 & 528 & 0.2 & 0.4 & 526 & $50.8 \%$ & $3.30(3.26,3.34)$ \\
\hline Total $(95 \% \mathrm{CI})$ & & & 584 & & & 579 & $100.0 \%$ & $2.86(1.98,3.74)$ \\
\hline
\end{tabular}

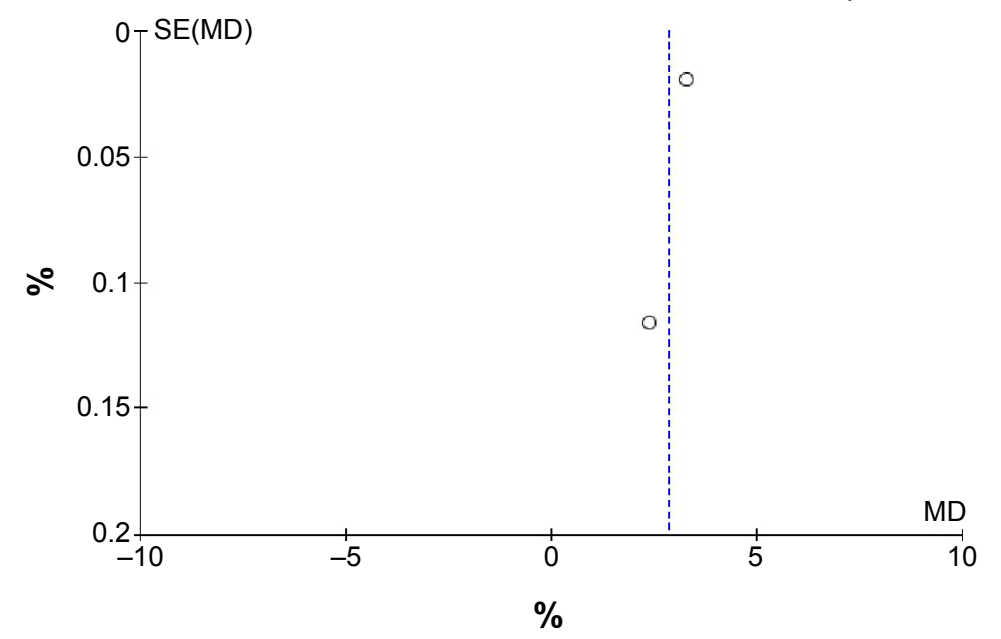

Figure 2 Eldecalcitol increases lumbar BMD, as compared with placebo.

Abbreviations: SD, standard deviation; $\mathrm{Cl}$, confidence interval; BMD, bone mineral density; $d f$, degree of freedom; MD, mean difference; SE, standard error. 


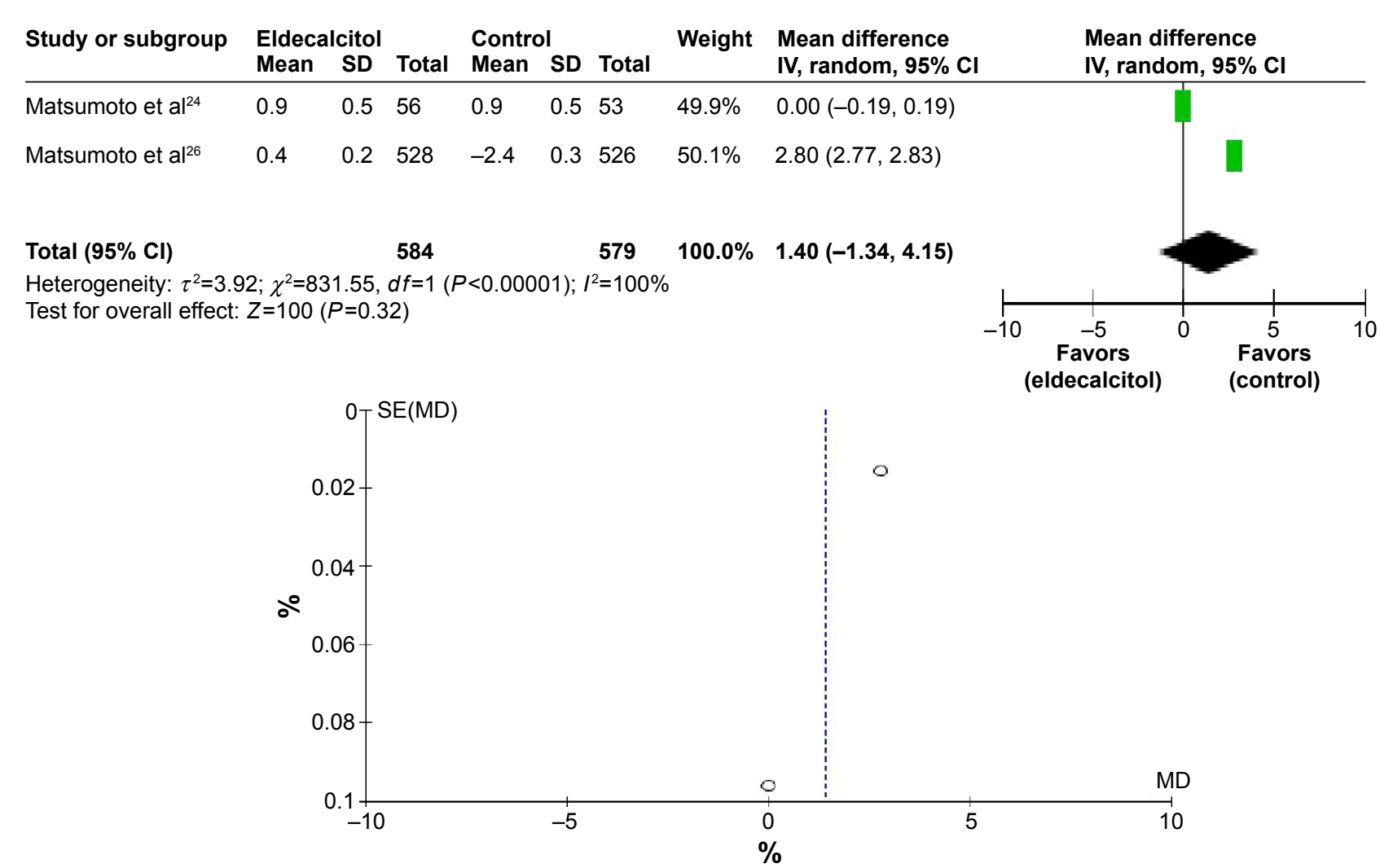

Figure 3 Eldecalcitol has no effect on hip BMD, as compared to placebo.

Abbreviations: SD, standard deviation; $\mathrm{Cl}$, confidence interval; BMD, bone mineral density; $\mathrm{df}$, degree of freedom; MD, mean difference; SE, standard error.

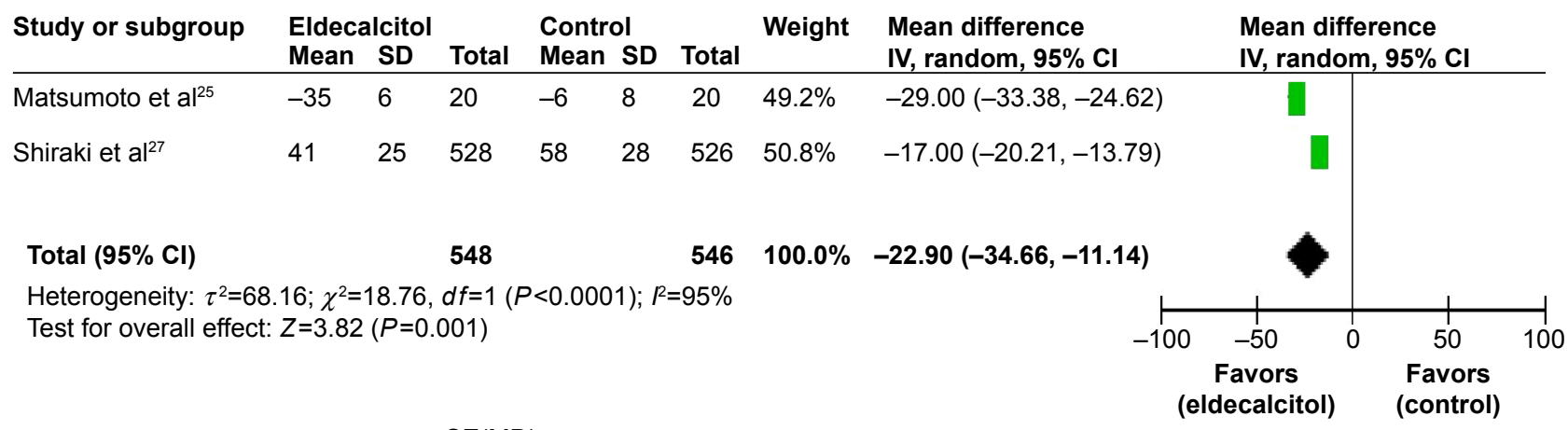

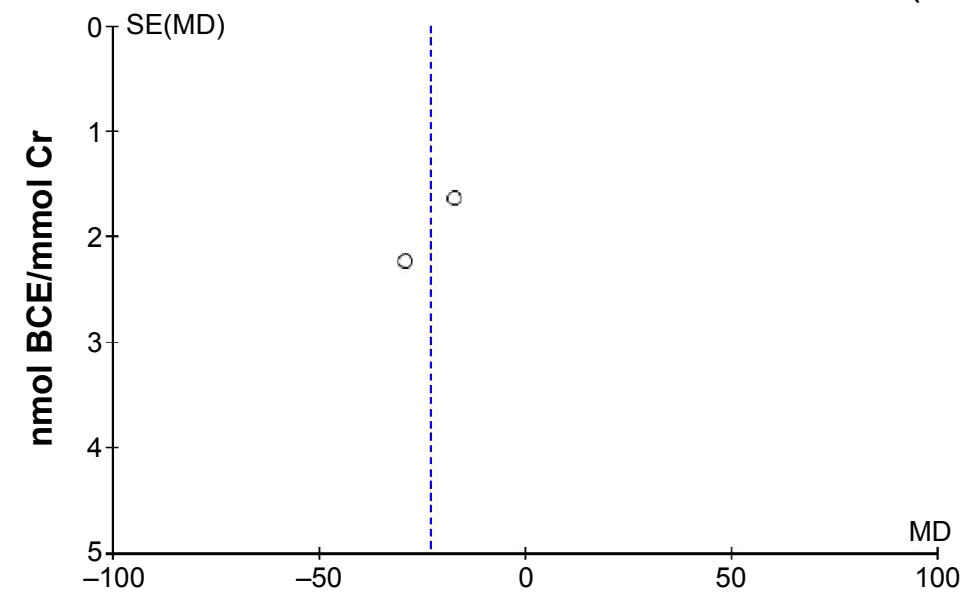

Figure 4 Eldecalcitol suppressed NTX to a greater degree in comparison with alfacalcidol.

Abbreviations: SD, standard deviation; Cl, confidence interval; NTX, type I collagen amino-terminal telopeptide; df, degree of freedom; MD, mean difference; SE, standard error. 
was found to do better than $1 \mu \mathrm{g}$ /day of alfacalcidol in lowering serum BALP $(26 \pm 9$ vs $32 \pm 11 \mathrm{U} / \mathrm{L}, P<0.05)$ and PINP $(42 \pm 15$ vs $59 \pm 23 \mathrm{ng} / \mathrm{mL}, P<0.05) .{ }^{27}$

The third trial also compared the efficacy of $0.75 \mu \mathrm{g} /$ day of edecalcitol and $1 \mu \mathrm{g}$ /day of alfacalcidol in preventing osteoporotic fractures. ${ }^{28}$ Their post hoc analyses found that ELD treatment reduced the incidence of osteoporotic fractures defined by the WHO Technical Report more than alfacalcidol treatment (18.6\% vs $25.2 \%$; hazard ratio [HR], $0.70 ; 95 \%$ CI, 0.54-0.93). ELD also decreased the incidence of major osteoporotic fractures defined in the WHO Fracture Risk Assessment Tool (FRAX) more than alfacalcidol (11.1\% vs $16.3 \%$; HR, 0.66; $95 \%$ CI, 0.46-0.94).

The third trial further evaluated the effects of ELD on the spinal location of incident vertebral fractures, the severity of the fractures, and the changes in health-related quality of life (HRQOL) in comparison to those of alfacalcidol. ${ }^{29}$ The incidence of vertebral fractures was evaluated based on the location on the spine (upper T4-T10; lower T11-L4). The severity of vertebral fractures was determined by using a semiquantitative method, and the change in HRQOL was analyzed by using the Medical Outcomes Study Short-Form 36-Item questionnaire. The data showed that the incidence of vertebral fracture at the lower spine was less in the ELD group than in the alfacalcidol group $(P=0.029)$, and the incidence of severe vertebral fracture (grade 3 ) was less in the ELD group than the alfacalcidol group $(3.8 \%$ vs $6.7 \%, P=0.036)$. The data also revealed that both ELD and alfacalcidol improved HRQOL in osteoporotic patients. Although no significant differences in each HRQOL scores were observed between ELD and alfacalcidol during the observational period, overall improvement from baseline of HRQOL scores was clearly observed in the ELD group. In summary, compared to alfacalcidol, ELD could further reduce the incidences of lower spinal vertebral fractures and severe vertebral fractures. Furthermore, daily treatment with ELD was effective in improving HRQOL.

\section{Conclusion and discussion}

Vitamin D has long been regarded as a fundamental part of the prevention and treatment of osteoporosis. However, findings from observational studies showed inconsistent associations between BMD and vitamin D status. ${ }^{30,31}$ In fact, result from a recent meta-analysis of trials of vitamin $\mathrm{D}$ alone failed to show an association between supplementation and BMD. ${ }^{32}$ In addition, results from meta-analyses of trials of vitamin D alone failed to show an association between supplementation and fracture prevention. ${ }^{33,34}$ Nevertheless, the active form of vitamin D, calcitriol, and its prodrug, alfacalcidol, have shown activities to increase BMD and reduce the risk of vertebral and nonvertebral fractures, and have been approved for the treatment of osteoporosis in Japan. Our current meta-analysis revealed that ELD was superior to alfacalcidol in suppressing NTX level. In fact, as was found in a recent clinical trial, alfacalcidol increased the lumbar BMD but decreased hip BMD, whereas ELD increased both the lumber and hip BMDs. ${ }^{26}$ Our review of data from multiple clinical trials also showed that ELD performed better than alfacacidol in reducing incidence of severe vertebral fractures and suppressing BTMs, including serum BALP and PINP. Put together, current data have provided strong support to the notion that active vitamin D analogs could be tailored in ways to improve their beneficial effects while limiting the unwanted activities for treatment of diseases such as osteoporosis.

Other widely used drugs for osteoporosis include bisphosphonates, calcitonin, estrogen hormone, estrogen agonist/ antagonist, PTH, and RANK ligand (RANKL)/RANKL inhibitor. Given the fact that the active analogs of vitamin D exert their pharmacological activities by mechanisms different from those of other current drugs, combination therapy with active vitamin $\mathrm{D}$ and other categories can be expected to offer more choices in osteoporosis treatment in the future. In fact, two such clinical trials have been conducted recently. In one trial, the effects of bisphosphonate alone on biochemical markers and muscle power in comparison with those of bisphosphonate combined with ELD were studied in postmenopausal women with osteoporosis. During the 6-month treatment period, while both treatments significantly reduced BTMs serum BALP and urinary NTX to a similar degree, combination therapy improved five-repetition chair-rising time to a significantly greater degree than monotherapy with bisphosphonate. ${ }^{35}$ In another trial, efficacy of combination of alendronate and ELD was compared to that of combination of alendronate plus vitamin D and calcium in patients with primary osteoporosis. The results showed that alendronate combined with ELD was more effective in reducing the BTMs, including serum type I collagen C-telopeptide (sCTX), urine NTX, tartrate-resistant acid phosphatase 5b (TRACP-5b), BALP, and PINP and increasing the femoral neck BMD compared to alendronate treatment with vitamin D3 and calcium. ${ }^{36}$ Therefore, ELD has shown promising potential in treatment of osteoporosis when combined with other existing antiosteoporotic drugs, and further exploration of ELD-based combination therapy is warranted.

Due to multiple reasons, our meta-analysis results should be interpreted and translated into clinical practice with caution. First of all, limited number of trials have been completed so 
far; second, risk of bias may be high in some of these trials; third, all trials have been conducted on Japanese patients, and the applicability of these data to other ethnic groups needs to be investigated; finally, data for some parameters were reported in different format in different trials (eg, interquartile in one study, but means in another study), making them less accurate and less comparable. However, over all clinical data that have been generated so far strongly support ELD as an effective treatment option for osteoporosis. Further clinical studies will surely reveal the full potential of ELD either as monotherapy or combination therapy for osteoporosis.

\section{Disclosure}

The authors report no conflicts of interest in this work.

\section{References}

1. Consensus development conference: diagnosis, prophylaxis, and treatment of osteoporosis. Am J Med. 1993;94:646-650.

2. Funck-Brentano T, Biver E, Chopin F, et al. Clinical utility of serum bone turnover markers in postmenopausal osteoporosis therapy monitoring: a systematic review. Semin Arthritis Rheum. 2011;41:157-169.

3. Kunchur R, Need A, Hughes T, Goss A. Clinical investigation of C-terminal cross-linking telopeptide test in prevention and management of bisphosphonate-associated osteonecrosis of the jaws. J Oral Maxillofac Surg. 2009;67:1167-1173.

4. Lewiecki EM. Benefits and limitations of bone mineral density and bone turnover markers to monitor patients treated for osteoporosis. Curr Osteoporos Rep. 2010;8:15-22.

5. Okamoto K, Inaba M, Furumitsu Y, et al. Beneficial effect of risedronate on arterial thickening and stiffening with a reciprocal relationship to its effect on bone mass in female osteoporosis patients: a longitudinal study. Life Sci. 2010;87:686-691.

6. van Leeuwen JP, van Driel M, van den Bemd GJ, Pols HA. Vitamin D control of osteoblast function and bone extracellular matrix mineralization. Crit Rev Eukaryot Gene Expr. 2001;11:199-226.

7. Langub MC, Reinhardt TA, Horst RL, Malluche HH, Koszewski NJ. Characterization of vitamin D receptor immunoreactivity in human bone cells. Bone. 2000;27:383-387.

8. Mee AP, Hoyland JA, Braidman IP, Freemont AJ, Davies M, Mawer EB. Demonstration of vitamin $\mathrm{D}$ receptor transcripts in actively resorbing osteoclasts in bone sections. Bone. 1996;18:295-299.

9. Brommage R, DeLuca HF. Evidence that 1,25-dihydroxyvitamin D3 is the physiologically active metabolite of vitamin D3. Endocr Rev. 1985; 6:491-511.

10. Khosla S. Minireview: the OPG/RANKL/RANK system. Endocrinology. 2001;142:5050-5055.

11. DeLuca HF. Overview of general physiologic features and functions of vitamin D. Am J Clin Nutr. 2004;80:1689S-1696S.

12. Nishii $Y$. Active vitamin $D$ and its analogs as drugs for the treatment of osteoporosis: advantages and problems. J Bone Miner Metab. 2002; 20:57-65.

13. Nishii Y. Rationale for active vitamin $\mathrm{D}$ and analogs in the treatment of osteoporosis. J Cell Biochem. 2003;88:381-386.

14. Nishii $Y, O k a n o T$. History of the development of new vitamin $D$ analogs: studies on 22-oxacalcitriol (OCT) and 2beta-(3-hydroxypropoxy) calcitriol (ED-71). Steroids. 2001;66:137-146.

15. Bouillon R, Okamura WH, Norman AW. Structure-function relationships in the vitamin D endocrine system. Endocr Rev. 1995;16:200-257.

16. Okuda N, Takeda S, Shinomiya K, et al. ED-71, a novel vitamin D analog, promotes bone formation and angiogenesis and inhibits bone resorption after bone marrow ablation. Bone. 2007;40:281-292.
17. Orimo H, Nakamura T, Hosoi T, et al. Japanese 2011 guidelines for prevention and treatment of osteoporosis - executive summary. Arch Osteoporos. 2012;7:3-20.

18. Gallagher JC, Fowler SE, Detter JR, Sherman SS. Combination treatment with estrogen and calcitriol in the prevention of age-related bone loss. J Clin Endocrinol Metab. 2001;86:3618-3628.

19. Shikari M, Kushida K, Yamazaki K, Nagai T, Inoue T, Orimo H. Effects of 2 years' treatment of osteoporosis with 1 alpha-hydroxy vitamin D3 on bone mineral density and incidence of fracture: a placebo-controlled, double-blind prospective study. Endocr J. 1996;43:211-220.

20. O’Donnell S, Moher D, Thomas K, Hanley DA, Cranney A. Systematic review of the benefits and harms of calcitriol and alfacalcidol for fractures and falls. J Bone Miner Metab. 2008;26:531-542.

21. Papadimitropoulos E, Wells G, Shea B, et al. Meta-analyses of therapies for postmenopausal osteoporosis. VIII: meta-analysis of the efficacy of vitamin D treatment in preventing osteoporosis in postmenopausal women. Endocr Rev. 2002;23:560-569.

22. Ringe JD, Schacht E. Potential of alfacalcidol for reducing increased risk of falls and fractures. Rheumatol Int. 2009;29:1177-1185.

23. Uchiyama $Y$, Higuchi $Y$, Takeda S, et al. ED-71, a vitamin D analog, is a more potent inhibitor of bone resorption than alfacalcidol in an estrogendeficient rat model of osteoporosis. Bone. 2002;30:582-588.

24. Matsumoto T, Miki T, Hagino H, et al. A new active vitamin D, ED-71, increases bone mass in osteoporotic patients under vitamin D supplementation: a randomized, double-blind, placebo-controlled clinical trial. J Clin Endocrinol Metab. 2005;90:5031-5036.

25. Matsumoto T, Takano T, Yamakido S, Takahashi F, Tsuji N. Comparison of the effects of eldecalcitol and alfacalcidol on bone and calcium metabolism. J Steroid Biochem Mol Biol. 2010;121:261-264.

26. Matsumoto T, Ito M, Hayashi Y, et al. A new active vitamin D3 analog, eldecalcitol, prevents the risk of osteoporotic fractures - a randomized, active comparator, double-blind study. Bone. 2011;49:605-612.

27. Shiraki M, Saito H, Matsumoto T. Eldecalcitol normalizes bone turnover markers regardless of their pre-treatment levels. Curr Med Res Opin. 2012;28:1547-1552.

28. Nakamura T, Takano T, Fukunaga M, Shiraki M, Matsumoto T. Eldecalcitol is more effective for the prevention of osteoporotic fractures than alfacalcidol. J Bone Miner Metab. 2013;31:417-422.

29. Hagino H, Takano T, Fukunaga M, Shiraki M, Nakamura T, Matsumoto T. Eldecalcitol reduces the risk of severe vertebral fractures and improves the health-related quality of life in patients with osteoporosis. J Bone Miner Metab. 2013;31:183-189.

30. Bischoff-Ferrari HA, Kiel DP, Dawson-Hughes B, et al. Dietary calcium and serum 25-hydroxyvitamin D status in relation to BMD among US adults. J Bone Miner Res. 2009;24:935-942.

31. Marwaha RK, Tandon N, Garg MK, et al. Bone health in healthy Indian population aged 50 years and above. Osteoporos Int. 2011;22: 2829-2836.

32. Reid IR, Bolland MJ, Grey A. Effects of vitamin D supplements on bone mineral density: a systematic review and meta-analysis. Lancet. 2014;383:146-155.

33. DIPART (Vitamin D Individual Patient Analysis of Randomized Trials) Group. Patient level pooled analysis of 68500 patients from seven major vitamin D fracture trials in US and Europe. BMJ. 2010;340:b5463.

34. Avenell A, Gillespie WJ, Gillespie LD, O'Connell D. Vitamin D and vitamin D analogues for preventing fractures associated with involutional and post-menopausal osteoporosis. Cochrane Database Syst Rev. 2009:CD000227.

35. Iwamoto J1, Sato Y2. Eldecalcitol improves chair-rising time in postmenopausal osteoporotic women treated with bisphosphonates. Ther Clin Risk Manag. 2014;10:51-59.

36. Sakai A, Ito M, Tomomitsu T, et al; e-ADVANCED Study Group. Efficacy of combined treatment with alendronate (ALN) and eldecalcitol, a new active vitamin D analog, compared to that of concomitant ALN, vitamin D plus calcium treatment in Japanese patients with primary osteoporosis. Osteoporos Int. 2015;26(3):1193-1202. 


\section{Publish your work in this journal}

Drug Design, Development and Therapy is an international, peerreviewed open-access journal that spans the spectrum of drug design and development through to clinical applications. Clinical outcomes, patient safety, and programs for the development and effective, safe, and sustained use of medicines are a feature of the journal, which has also been accepted for indexing on PubMed Central. The manuscript management system is completely online and includes a very quick and fair peer-review system, which is all easy to use. Visit http://www.dovepress.com/testimonials.php to read real quotes from published authors.

Submit your manuscript here: http://www.dovepress.com/drug-design-development-and-therapy-journal 